\title{
Perfil demográfico e de morbimortalidade de pacientes que internam por insuficiência cardíaca aguda descompensada em hospital terciário brasileiro
}

\author{
Wajner, A.; Fernandes, E.O.; Fensterseifer, G.S.; Landenberger, T.; Waldemar, \\ F.S.; Pereira, G.A.R.; Polanczyk, C.A.;
}

Apresentador: Tiago Landenberger

\section{Resumo}

Introdução: A insuficiência cardíaca (IC) é uma doença de alta prevalência, mas e segundo dados do DATASUS, representa a maior causa cardiovascular de morbidade hospitalar. Apesar disso, na América Latina são escassos os estudos sobre o impacto gerado pela IC em termos de hospitalizações e morbimortalidade. Assim, os objetivos do nosso estudo são descrever o perfil demográfico e indicadores de morbimortalidade de pacientes que internam por IC aguda descompensada (ICad) em hospital terciário público brasileiro. Métodos: Realizou-se estudo de coorte histórico com utilização de dados secundários com 2069 pacientes que internaram com o diagnóstico de IC estabelecido através do registro pelo médico assistente (ma) do Índice de Comorbidade de Charlson (ICCharlson) preenchido compulsoriamente no prontuário eletrônico no momento da internação e alta hospitalar no período de 2009-2010. Destes, 28,8\% tiveram como causa principal de internação a ICad que foi definida pelo preenchimento pelo ma do CID principal correspondente a IC. A coleta de dados foi realizada através da revisão de prontuários eletrônicos. Resultados: Foram coletadas 595 pacientes com diagnóstico de ICad. A amostra apresenta 50,8\% de homens, 82,7\% de brancos, idade média de 68,81 anos (dp 13,48) e a maioria era procedente de Porto Alegre (63,2\%). Os pacientes internaram no serviço de cardiologia em $62,9 \%$. A mediana do ICCharlson foi de 5,0 (IQ 25-75\%:4-7), sendo as comorbidades mais freqüentes diabete melito $(28,9 \%)$, infarto agudo do miocárdio (19,5\%), doença pulmonar crônica $(18,2 \%)$ e doença renal $(13,8 \%)$. Ao avaliar-se dados ecocardiográficos, do total da amostra, observou-se $53 \%$ com Fração de Ejeção (FE) <40\%, 38,1\% com Fej $>40 \%$, sendo que $8,9 \%$ não apresentavam relato de FE ou não tinha ecocardiografia. Constatou-se em 29,6\% alteração segmentar, em 43,4\% alteração difusa e em 40,7\% hipertrofia de câmaras cardíacas. Com relação aos aspectos hospitalares, a mediana de internação foi de 13 dias (IQ 25- 75\%:9-20), 14,8\% dos pacientes apresentaram passagem pela UTI, sendo a mortalidade hospitalar de $18,8 \%$ e reinternação neste hospital em 30 dias de 4,2\%. Conclusão: Demonstrou- se, em um hospital terciário brasileiro, elevado grau de morbimortalidade da IC, com pacientes apresentando comorbidades e mortalidade superior à literatura internacional. Necessita-se realizar mais estudos para compreendermos o real impacto sócio-econômico da IC no sistema de saúde brasileiro e na sociedade em geral.

\section{Referência:}

Wajner, A.; Fernandes, E.O.; Fensterseifer, G.S.; Landenberger, T.; Waldemar, F.S.; Pereira, G.A.R.; Polanczyk, C.A.;. Perfil demográfico e de morbimortalidade de pacientes que internam por insuficiência cardíaca aguda descompensada em hospital terciário brasileiro. In: II Congresso Brasileiro de Medicina Hospitalar - II CBMH [= Blucher Medical Proceedings, vol.1, num.5] São Paulo: Editora Blucher, 2014. p.97

DOI 10.5151/medpro-II-cbmh-096 